\title{
Species Richness at Continental Scales Is Dominated by Ecological Limits*
}

\author{
Daniel L. Rabosky ${ }^{1 \dagger}$ and Allen H. Hurlbert ${ }^{2}$ \\ 1. Museum of Zoology and Department of Ecology and Evolutionary Biology, University of Michigan, Ann Arbor, Michigan 48103; \\ 2. Department of Biology and Curriculum for the Environment and Ecology, University of North Carolina, Chapel Hill, North Carolina \\ 27599
}

ABSTRACT: Explaining variation in species richness among provinces and other large geographic regions remains one of the most challenging problems at the intersection of ecology and evolution. Here we argue that empirical evidence supports a model whereby ecological factors associated with resource availability regulate species richness at continental scales. Any large-scale predictive model for biological diversity must explain three robust patterns in the natural world. First, species richness for evolutionary biotas is highly correlated with resource-associated surrogate variables, including area, temperature, and productivity. Second, species richness across epochal timescales is largely stationary in time. Third, the dynamics of diversity exhibit clear and predictable responses to mass extinctions, key innovations, and other perturbations. Collectively, these patterns are readily explained by a model in which species richness is regulated by diversity-dependent feedback mechanisms. We argue that many purported tests of the ecological limits hypothesis, including branching patterns in molecular phylogenies, are inherently weak and distract from these three core patterns. We have much to learn about the complex hierarchy of processes by which local ecological interactions lead to diversity dependence at the continental scale, but the empirical evidence overwhelmingly suggests that they do.

Keywords: speciation, extinction, diversity dependence, macroecology, equilibrium.

\section{Introduction: For Ecological Limits}

For decades, biologists have debated the relative contributions of equilibrium and nonequilibrium processes to large-scale patterns of species richness. Addressing this issue remains one of the most important challenges in ecology and evolutionary biology because it has broad implications for understanding the history of diversity, the processes that generate diversity, and the future of diver-

* An earlier version of this article was presented as part of the American Society of Naturalists debate, which was held at the ASN stand-alone meeting at Asilomar, California, in January 2014.

† Corresponding author; e-mail: drabosky@umich.edu.

Am. Nat. 2015. Vol. 185, pp. 572-583. (C) 2015 by The University of Chicago. 0003-0147/2015/18505-55789\$15.00. All rights reserved.

DOI: $10.1086 / 680850$ sity in a rapidly changing world. In this perspective, we assert that species richness at continental scales is largely equilibrial and dominated by ecological limits. In contrast, our opponents in this debate (Harmon and Harrison 2015) propose that species richness at the largest spatial scales is nonequilibrial and that ecological limits are either nonexistent or unimportant relative to other processes that influence the dynamics of speciation and extinction.

We argue that the empirical evidence is consistent with a theory of species richness whereby diversity-dependent feedback mechanisms regulate the number of species within large landmasses. This idea is simultaneously a statement about a process, its generality, and the spatiotemporal scale to which it applies. By "continental scales," we restrict the scope of our arguments to landmasses that are sufficiently large that the majority of standing diversity is derived from in situ speciation and not immigration from other landmasses ("mainlands," in the terminology of Rosenzweig 1995). One can replace the word "continental" with "provincial" to apply to marine faunas to much the same effect (Rosenzweig 1995).

In its simplest form, the ecological limits hypothesis (ELH) asserts (1) that species richness at biogeographic scales exists in a state of dynamic equilibrium, (2) that this equilibrium results from diversity dependence of speciation and/or extinction rates, and (3) that diversity dependence of evolutionary rates results from constraints on total resource availability. Thus, the "ecological limit" is the constraint on total resource availability, not a fixed limit on the number of species that can occur in a system. By arguing that continental systems are "dominated" by ecological limits, we claim that most of the variance in species richness among all such geographic regions can be attributed to ecological limits. As such, species richness should largely be predictable from knowledge of general properties of a system that reflect total resource availability. We do not believe that diversity equilibria can exist in a meaningful sense without ecological limits on resources. In the absence of such limits, there is little reason to postulate the 
diversity dependence of speciation and extinction that results in equilibrial dynamics. Even MacArthur and Wilson's (1963) theory of island biogeography invokes ecological limits in order to predict positive relationships between island size and species richness (see below; Rosenzweig 1995).

The ELH has a long history, and it is not our intention to review this literature here. Systems that are governed by ecological limits are sometimes described as "saturated" or possessing "carrying capacities." These terms invite confusion because they can be taken to imply that species diversity is static or that there are a fixed number of ecological niches. However, strong regulation by ecological limits implies neither fixed numbers of niches nor static diversity. Under ecological limits, the number of species in a system should be the outcome of a stochastic process with a mean value determined by total resource availability. The quantity of resources can itself fluctuate through time, perhaps through secular changes in geochemical processes (Vermeij 1995; Vermeij and Roopnarine 2013), changes in continental shelf area (Peters 2005), or through episodic key innovations that increase the capacity of organisms to use resources (Boyce et al. 2009). Such perturbations to resource availability should facilitate periodic expansions in species richness, potentially cascading through other trophic levels (Boyce and Lee 2010; Bush and Bambach 2011; Allmon and Martin 2014).

We first describe a simple model illustrating the logic of the ELH. The model is agnostic with respect to specific population-level mechanisms but provides a heuristic tool for understanding how ecological limits can generate variation in species richness among clades and regions. We then describe three major biodiversity patterns that are consistent with ecological limits yet difficult to explain in their absence. We then discuss other forms of evidence that are variously interpreted as for and against the ecological limits hypothesis.

\section{A Phenomenological Model for Ecological Limits}

An argument for ecological limits is effectively an argument for the diversity dependence of mean per-lineage net diversification rates. If per-lineage speciation rates decrease and/or per-lineage extinction rates increase with increasing richness, then richness will ultimately approach and fluctuate about the equilibrial value where those rates are equal (fig. 1). The dynamic balance between speciation and extinction is based on the colonization-extinction dynamics presented in MacArthur and Wilson's $(1963,1967)$ theory of island biogeography as well as a subsequent modification for mainland systems (MacArthur 1969; Rosenzweig 1975; Brown et al. 2001). Diversity equilibrium in the MacArthurWilson model emerges, in part, because of ecological limits on the total number of individuals that can occupy an island. Per-lineage extinction rates in the model rise as a function of the number of species on an island because mean population sizes per species decline as richness increases. Hence, for a given level of species richness, extinction rates are lower on large islands than small islands, leading to greater equilibrium diversities on large islands. Although purely phenomenological, the simple model in figure 1 illustrates two points that are often overlooked or misunderstood in the debate on ecological limits, and it can accommodate a wide range of specific mechanisms for diversity dependence.

First, the model clarifies the role resource availability plays in determining the equilibrium richness of a region relative to other factors. The finite nature of resources is the ecological limit for a region that results in a zero-sum game and the ensuing dynamic equilibrium between speciation and extinction (Van Valen 1976; Hubbell 2001; Hurlbert and Stegen 2014b). Variation in resource availability is expected to shift equilibrial richness in a manner analogous to the effects of area in the theory of island biogeography, by influencing the functional relationship between the extinction rate and species richness (Wright 1983; fig. 1B).

However, changes in the shape of the relationships between diversification and species richness can influence realized equilibrium richness, regardless of the underlying pool of resources. This observation implies immediately that regions with the same resource base can have different equilibrium diversities (fig. $1 C, S_{1}$ vs. $S_{2}$ ), if they differ in processes that affect rates of speciation and extinction. For example, if one region had increased "background" speciation rates due to increased topographical complexity (Cracraft 1985; Badgley 2010) or increased temperature-driven mutation rates (Allen et al. 2006; Gillooly and Allen 2007) relative to another region, then it would be expected to support more species at equilibrium (fig. 1C). Similarly, clades might differ in key traits that make them more or less prone to speciation (or extinction), suggesting that the phylogenetic makeup of a biota will affect equilibrial species richness $\left(S_{\text {eq }}\right)$ as well.

Second, the model demonstrates how geographic regions can differ in their equilibrium diversity even if all regions have identical speciation and extinction rates in the present day (fig. 1D). This point is particularly important because it means that finding identical evolutionary rates for regions that vary in diversity is uninformative about the role of equilibrium processes in generating that diversity. The causes of differential diversity must be sought not only in the rates themselves but also in the derivatives of these rates with respect to species richness (Rosenzweig 1995).

This phenomenological model is consistent with a broad range of proposed mechanisms that result in diversity- 

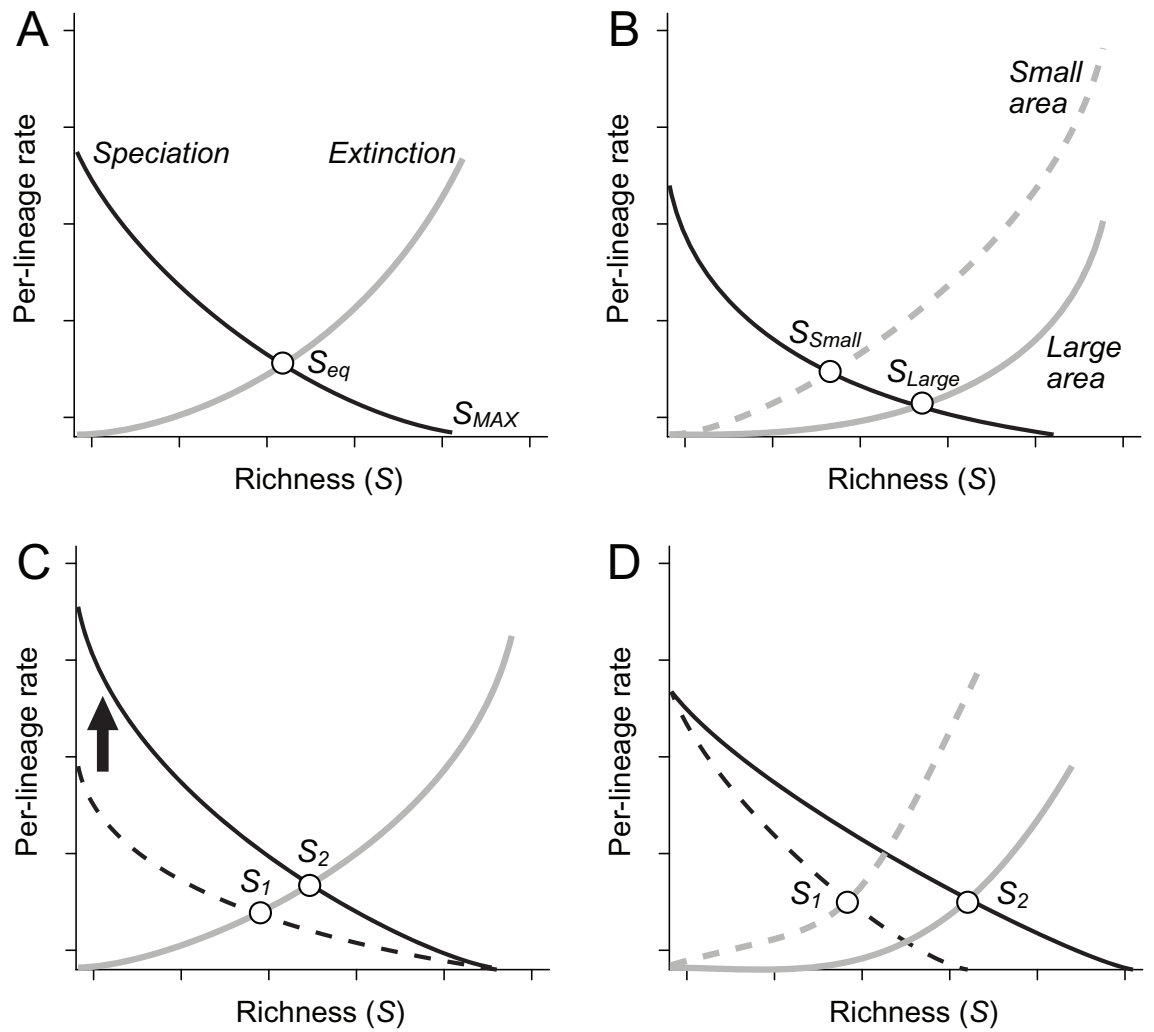

Figure 1: $A$, Equilibrium model for the assembly of continental biotas. In this example, speciation rates decline and extinction rates increase as a function of species richness; the intersection between these curves is the equilibrial species richness $\left(S_{\text {eq }}\right)$. As in MacArthur and Wilson (1967), extinction rates should be low when a geographic region is occupied by a few species with large population sizes and high when a region is occupied by many species with smaller population sizes. Here $S_{\mathrm{MAX}}$ is the theoretical maximum species richness that would be obtained by speciation in the absence of any extinction. The form of the curves shown here is arbitrary, and equilibrium will emerge even in the absence of diversity-dependent speciation, provided that per capita population sizes decline with increasing species richness. $B$, Regional area and/or resource availability should influence the form of the relationship between extinction and species richness, with the expectation that larger areas with more resources will have greater equilibrial species richness. $C, \mathrm{~A}$ factor that increases the baseline rate of speciation or immigration (arrow) will increase equilibrial species richness (from $S_{1}$ to $S_{2}$ ), even if the resource pool remains unchanged. Geographic regions with identical resources can thus vary in their equilibrium richness values as a function of regional or clade-specific factors that affect speciation or immigration rates. $D$, Geographic regions can vary in equilibrium species richness even if speciation and extinction rates are exactly equal. Hence, a finding that speciation rates are similar between geographic regions that differ in richness provides no evidence for a nonequilibrium model of diversity accumulation.

dependent dynamics of speciation and extinction. Sepkoski's (1978) pioneering study on diversity dependence in the fossil record provided a lucid discussion of populationlevel mechanisms by which species interactions could influence speciation and extinction probabilities. More recent work has expanded on the consequences of local ecological interactions for species diversification via their emergent effects on population dynamics and geographic range size (Ricklefs and Bermingham 2007; Price 2010; Rabosky 2013; Hurlbert and Stegen 2014b). Geographic range size is negatively related to extinction risk (Manne et al. 1999; Payne and Finnegan 2007; Harnik et al. 2012) and may be positively correlated with opportunities for allopatric speci- ation (Mayr 1963; Price 2008; Kisel and Barraclough 2010). To the extent that resource-mediated interactions between species limit geographic distributions across continentalscale landscapes (Price and Kirkpatrick 2009; Sexton et al. 2009; Pigot and Tobias 2013), such interactions can influence the dynamics of speciation and extinction.

Much like island biogeography theory, the ecological limits hypothesis makes first-order predictions about pattern that can be tested with minimal knowledge of the underlying mechanisms. Specifically, the model predicts that species richness at biogeographic scales should in general correlate with total resources (or appropriate surrogate variables), that species richness should typically exist in a state 
of dynamic equilibrium, and that richness should respond predictably to perturbation.

\section{Ecological Limits Can Explain the "Big Three" Patterns of Diversity}

The predictions of the ecological limits hypothesis described above are strongly supported by three of the most striking large-scale spatiotemporal patterns in the structure and dynamics of biological diversity. First, most of the variation in species richness among major biogeographic regions can be explained by surrogate variables that reflect the total pool of resources available in a given geographic region. Second, species richness is generally constant in time, at least over timescales relevant to the establishment of diversity equilibria (e.g., epochal timescales; $<50$ million years). Third, the dynamics of species richness exhibit clear and predictable responses to perturbations, both positive and negative. Thus, mass extinctions are typically followed by recoveries, and major evolutionary innovations that increase the resource economy of the biota lead to increases in diversity. In isolation, each of these patterns may be consistent with nonequilibrial mechanisms of diversity regulation. Yet all three emerge immediately from the ecological limits hypothesis, while predictions of any single nonequilibrial model typically contradict one or more of these patterns.

\section{Species Richness Is Correlated with Area and Energy}

One of the most striking patterns in the global distribution of species richness is the correlation between the area of a region and its species richness (MacArthur and Wilson 1963). Area is, of course, a surrogate variable that scales with various measures of total resources (Wright 1983; Rosenzweig 1995). By virtue of an expanded resource base, larger areas can generally support more individuals than smaller areas; a consequence of this relationship is an increase in realized equilibrium diversities relative to smaller areas. At the scale of biotic provinces, area emerges consistently as the strongest single predictor of species diversity (Rosenzweig 1995), explaining up to $84 \%$ of the variation in terrestrial vertebrate richness among major zoogeographic regions (97\% for area plus a single climate parameter; Rosenzweig et al. 2012). At this spatial scale, the species-area relationship cannot reflect sampling effects, as we are considering entire biotas that were assembled largely by in situ diversification processes. Recent analyses at the level of biogeographic provinces have found strong effects of area, productivity, and temperature on vertebrate species richness. Although time-associated variables are highly correlated with the number of endemic species within regions (Jetz and Fine 2012), area and productivity remain the strongest predictors of total richness (fig. 2). For plants, energy- and productivity-associated variables consistently emerge as the strongest predictors of species richness (Currie 1991; Kreft and Jetz 2007). At continental to global scales, the relationship is unambiguous: every large-scale study reviewed by Gilman and Wright (2006) found a positive relationship between productivity and species richness. While ecological limits are not mutually exclusive with other hypotheses that might affect the shape of speciation and immigration curves, the strength of these correlations is consistent with the idea that ecological limits are the most important of these drivers.

Other hypotheses have been proposed to account for these patterns, including the kinetic effects of temperature on genetic divergence (Rohde 1992; Allen et al. 2002). While it is true that rates of speciation (or extinction) might vary systematically across geographic regions, this hypothesis predicts at best a weak relationship with area and other environmental factors, because any variation in the ages of clades among regions will decrease the relationship between diversification rate and richness. There has yet been no demonstration of a nonequilibrium process that can remotely approach the explanatory power of equilibrium models in accounting for evolutionary species-environment correlations. In the equilibrium framework described above, we noted that geographic variation in speciation or extinction rates can lead to differences in equilibrium diversities for regions with identical levels of resources (fig. 1C). This leads to a "weak" version of the ELH, whereby diversity is equilibrial but much of the variation in richness among geographic regions is nonetheless driven by regional variation in evolutionary rates. However, the general observation that species richness is highly correlated with resourceassociated variables suggests that, in general, equilibrium diversities are influenced more by resource availability than by these regional factors.

\section{Species Richness Is Not Characterized by Exponential Increase}

Diversity-independent models also predict high volatility in species richness: at any given point in time, species richness should be increasing exponentially or decreasing to zero. This volatility is rarely observed in the fossil record. This is not to say that diversity has not increased through time (clearly it has; see next section) but that the dynamics of diversity throughout most of the history of life on Earth argue in favor of a strongly regulated diversity-dependent process. The evidence for this pattern - and, in particular, against the idea that diversity ever shows exponential rises for any substantial durations of time-is too extensive to review adequately here. At global scales, marine biodiversity appears largely equilibrial across the entirety of the 

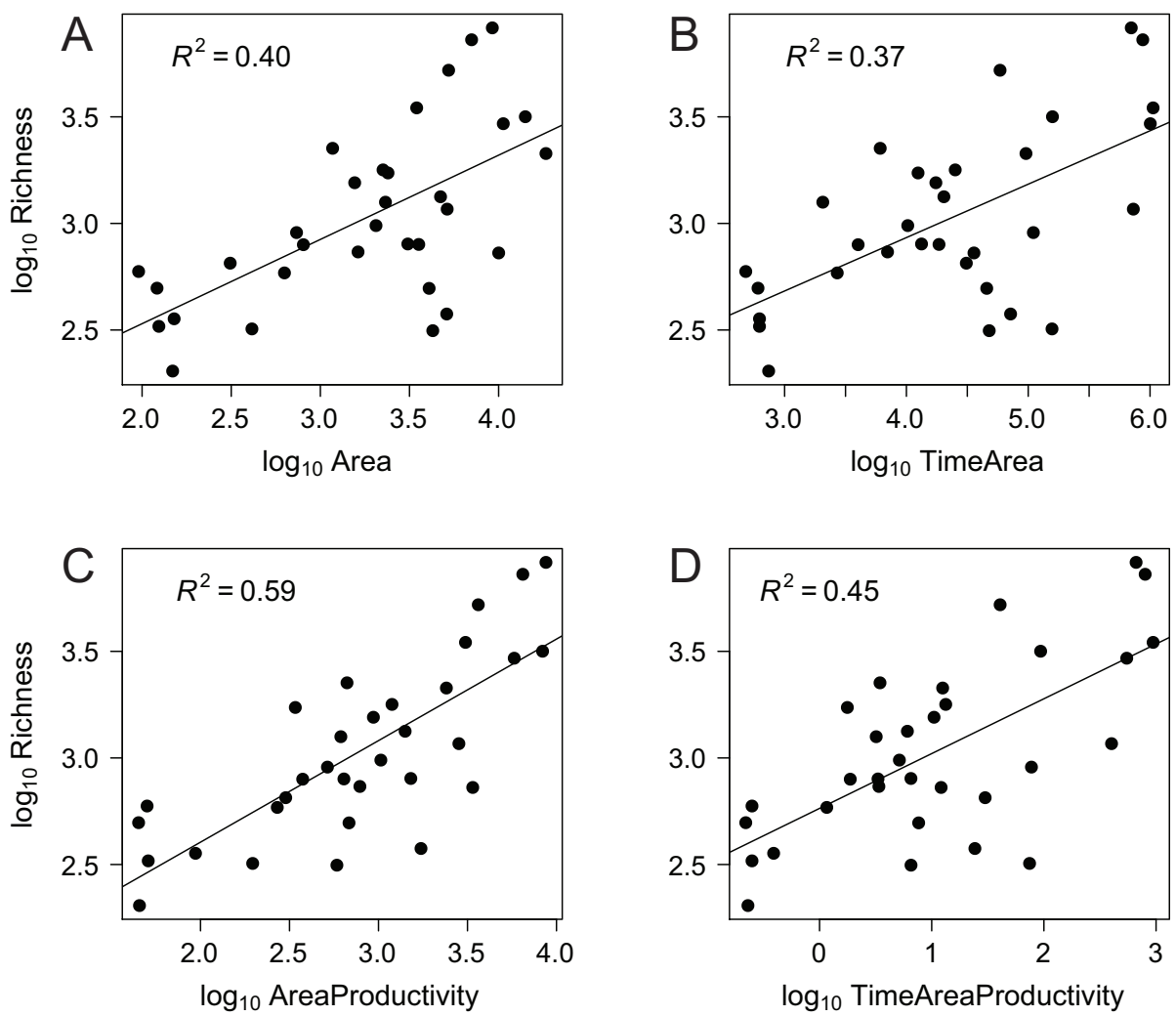

Figure 2: Relationship between total vertebrate species richness of 32 bioregions and bioregion area $(A)$, time-integrated bioregion area $(B)$, total contemporary bioregion productivity $(C)$, and total time-integrated bioregion productivity $(D)$. Contemporary productivity per unit area is a stronger predictor of total vertebrate richness than either area or time-integrated area. Data from Jetz and Fine (2012).

Phanerozoic, despite increases and decreases of diversity in response to the availability of shallow water and reef habitats (Alroy 2010a, 2010b). Similar patterns emerge for regional faunas using standardized methods of data collection. North American mammals collectively show largely equilibrial diversity dynamics (Alroy 2009), a pattern that holds for at least some individual subclades within mammals (Van Valkenburgh and Janis 1993; Liow and Finarelli 2014). Large-scale diversity trends are often coupled to climate-associated variables (Jaramillo et al. 2006), but these fluctuations in diversity are fully consistent with diversitydependent controls (Ezard et al. 2011).

Trends in local diversity through geological time can potentially provide strong tests of the equilibrial model (Wiens 2011), as local paleocommunities are less influenced by many confounding factors that compromise global or regional diversity curves (Bambach 1977). This test does not require independence of local and regional diversity but is simply based on the assumption that - under nonequilibrium, expansionist models - local diversity should continue to rise as new species are added to the regional species pool through speciation. The evidence from local com- munities is unambiguous: with the exception of episodic and transformative key innovations, local diversity is largely static through time, at least in the absence of severe environmental perturbations (e.g., Barry et al. 2002). For example, the evolution of angiosperms resulted in a pronounced rise in diversity within local assemblages (Knoll 1986; Lidgard and Crane 1990). However, it is incorrect to suggest that this shift in diversity is consistent with an overall exponential and unbounded rise in land plant diversity. Before the origin of angiosperms, local floras are characterized by stable diversity levels over much of the Paleozoic and Mesozoic (Knoll 1986). The pulse of angiosperm diversity did not lead to sustained exponential rise within local communities and was accompanied by concomitant declines in the diversity of gymnosperms and free-sporing plants (Lupia et al. 1999). Taphonomically matched samples from specific habitats have shown no change in floral richness over timescales that span several hundred million years (Wing and DiMichele 1995). Similar patterns are observed for the marine benthos, where episodic increases in species richness of marine invertebrates have undoubtedly occurred (Bambach 1977; Bush and Bambach 2011). However, these in- 
creases appear to have involved major expansions in ecological space associated with colonization of new habitats (e.g., the muddy benthos; Rosenzweig and Taylor 1980) or the evolution of fundamentally novel ecological strategies (Bambach et al. 2007; Bush and Bambach 2011).

\section{Species Richness Responds Predictably to Perturbations}

The ecological limits hypothesis makes two predictions about biotic responses to perturbations. First, negative perturbations - mass extinctions, in particular-should lead to diversity recoveries. Second, positive perturbations - increases in the resource base available to a biota-predict increases in species richness to stable but greater equilibrial levels. The diversity-independent model does not predict either of these patterns. Diversity recoveries entail a (relatively) rapid rise in species diversity in the aftermath of major extinction events, followed by the resumption of stationary dynamics showing little net change in richness. Diversity recoveries are documented for nearly all major extinction events and across a wide range of taxa (e.g., Krug and Patzkowsky 2004; Brayard et al. 2009). We are not arguing that recovery dynamics are simple, that rebounds occur instantaneously, or that postextinction ecosystems are functionally identical to pre-extinction ecosystems. Clearly, there is great complexity to the recovery process (Erwin et al. 1987; Erwin 2001): recovery may be associated with lag times (Chen and Benton 2012), ecological interactions may be restructured (Wagner et al. 2006), and postextinction replacement diversity may be phylogenetically distinct from the pre-extinction biota (Sallan and Coates 2010). But there is no question that recoveries typically occur, and this general phenomenon is difficult to explain in the absence of strong diversity-dependent controls.

A second type of perturbation occurs when intrinsic or extrinsic factors increase the resource base available to a biota. Clearly, the evolutionary invasion of new habitats has facilitated increases in species richness: the colonization of land, for example, led to a dramatic rise in global diversity across the Phanerozoic. However, other types of evolutionary innovations can permanently alter the resource constraints on biotas and lead to expansions of the equilibrial levels at which diversity is regulated (Vermeij and Roopnarine 2013). For example, the evolution of hydraulic features in angiosperm leaves more than doubled their photosynthetic capacity relative to gymnosperms and ferns (Boyce et al. 2009; Brodribb and Feild 2010; Jan de Boer et al. 2012). This event was a physiologically transformative key innovation that increased total energy flux through the biota and that, under the ELH, should have facilitated a global expansion of species richness. Although we are in the early stages of addressing the implications of these findings (Feild et al. 2011), the evolution of angio- sperm leaf hydraulics potentially facilitated the massive rise in flowering plant diversity that occurred in the late Cretaceous (Knoll 1986; Lupia et al. 1999). This and similar expansions in the energy economy of biotas appear to have had effects that are fully predicted by the ELH.

\section{Phylogenetic Data and the Ecological Limits Hypothesis}

In many ways, phylogenetic data have transformed our understanding of the diversification process. For the many groups of organisms that lack adequate fossil records, timecalibrated phylogenies of living species provide the only information we will ever have about variation in speciation rates through time and among lineages. Phylogenetic data will continue to be of fundamental importance in explaining why species richness varies so dramatically among different groups of organisms (Mitter et al. 1988; Coyne and Orr 2004). However, it is increasingly clear that there are limits to what can be inferred about diversity dynamics from phylogenetic data alone, and we agree with many points raised by Harmon and Harrison (2015). Below, we briefly highlight several reasons why phylogenetic tests of the ecological limits model are inherently weak, and-in particular - why they typically cannot be used to reject the ELH. A focus on phylogenetic diversification patterns can be a distraction from the three robust patterns outlined above, which are difficult to explain in the absence of ecological limits.

\section{Diversification Patterns in Time-Calibrated Molecular Phylogenies}

Molecular phylogenies frequently reveal evidence for decelerations in the rate of speciation through time during the course of evolutionary radiations, consistent with negative feedback between species richness and diversification (Rabosky and Lovette 2008; Etienne and Haegeman 2012). However, there are numerous caveats that apply to the interpretation of phylogenetic diversification patterns. We will not review these issues here, other than to note that phylogenetic estimates of diversification rates can be biased by taxon sampling, phylogeny reconstruction, and other factors (Revell et al. 2005; Cusimano and Renner 2010; Etienne and Rosindell 2012; Harmon and Harrison 2015). A number of confounding factors can create the impression that diversification has slowed through time (Rabosky 2009; Moen and Morlon 2014). In our view, the assumption that "early burst" patterns in molecular phylogenies are the only lineage accumulation patterns consistent with diversity dependence is conceptually flawed, because strictly equilibrial processes of diversity regulation are consistent with many patterns in phylogenetic trees that do not involve 
apparent slowdowns in speciation (Rabosky 2009). For example, the early burst signal is primarily relevant to early stages of an evolutionary radiation, and this signal will weaken and ultimately disappear for clades that have been at equilibrium for long periods of time (fig. 3).

\section{Positive "Time-for-Speciation" Relationship Is Consistent with Ecological Limits}

The effect of time on the species richness of geographic regions has long been recognized (Fischer 1960). If species richness is not regulated by diversity-dependent feedback mechanisms, and if speciation rates consistently exceed extinction rates, then diversity should generally increase through time. This logicism has led to the argument that if species richness of clades within a geographic region (or among regions: Stephens and Wiens 2003) is positively correlated with their age (or time within regions), then species richness cannot be equilibrial (Wiens 2011; Cornell 2013).
Thus, an observation that regions with greater species richness have had a longer history of occupancy, as inferred from phylogenetic data, would argue against the ecological limits hypothesis. There are several reasons for caution in analyzing the relationship between time and diversity. For example, it can be difficult to distinguish between asymptotic, bounded diversity trajectories (consistent with ecological limits) and exponential clade growth with high background extinction (Rabosky 2012).

Most importantly, a positive relationship between the ages of clades within regions and their species richness cannot reject the possibility that diversity is strictly equilibrial. In a spatial context, if a clade originates in a region of high resource availability and diversifies and disperses to adjacent regions with progressively fewer resources, even if diversification is governed by strictly equilibrial dynamics, a positive relationship is expected between the estimated time-within-region and species richness of the region (Hurlbert and Stegen 2014a). As such, the relationship between clade age (or time in a region) and species richness is not use-

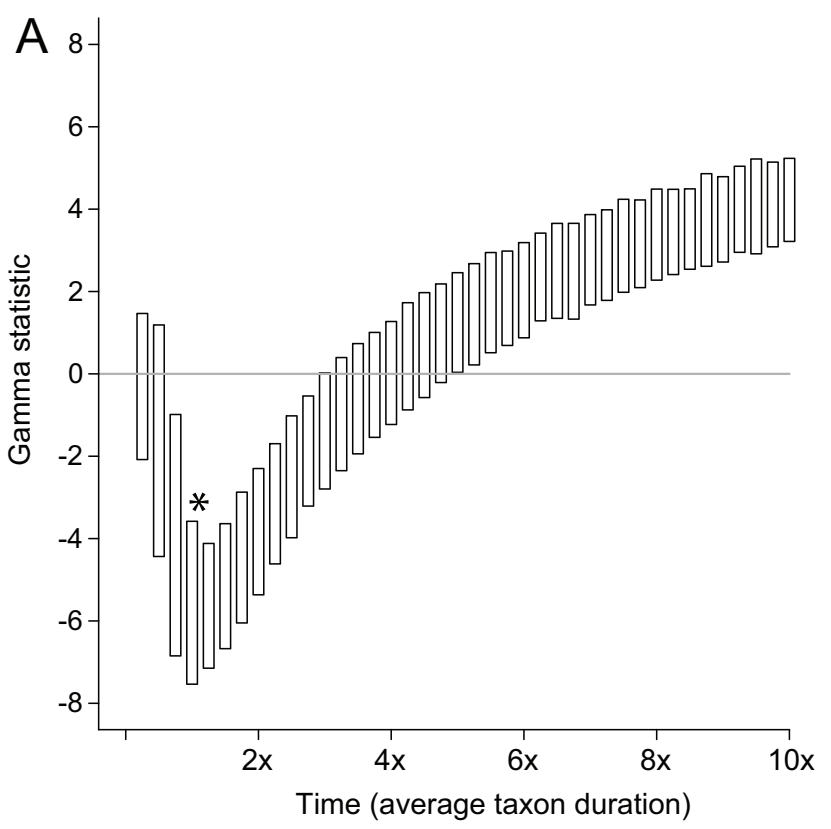

B

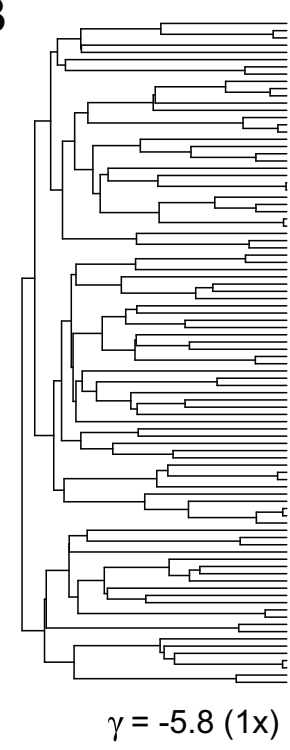

C

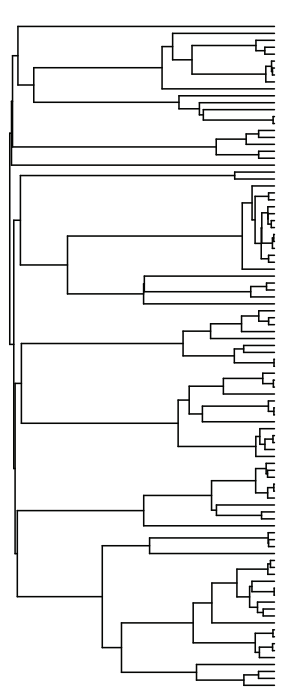

$\gamma=+4.9(10 x)$

Figure 3: $A$, Results of simulation illustrating that equilibrium diversification dynamics need not be associated with the "early burst" model of diversification. Five hundred phylogenies were simulated under a diversity-dependent speciation process with constant extinction, beginning with a single lineage and parameterized exactly as in figure 2 from Liow et al. (2010). Polygons give the medians and 90\% confidence intervals on the distribution of the gamma statistic $(\gamma)$ for reconstructed phylogenetic trees (e.g., with extinct species removed) at each time point. Values of $\gamma$ less than zero are suggestive of declining speciation rates through time. An asterisk indicates the point in simulation where $95 \%$ of simulated trees had reached their equilibrium diversity, mediated by equal speciation and extinction rates ( 90 lineages). Time is in units of expected taxon durations, which is simply the inverse of the extinction rate. The distribution of $\gamma$ is maximally negative at the point in time where equilibrium is first reached but rapidly becomes positive as lineage turnover erodes the signal of rapid speciation. $B$, A representative phylogenetic tree, pruned of extinct lineages, from the time when equilibrium was first reached $(1 \times$ taxon durations); note the concentration of early speciation events and negative $\gamma$. $C$, Representative phylogenetic tree $9 \times$ taxon durations after achieving equilibrium richness; $\gamma$ is positive, and most speciation events are clustered at the tips of the tree. Some residual signal of the initial speciation pulse is still present in $C$, but sufficient time (and/or fluctuations in population size) will ultimately eliminate this effect. 
ful for making inferences about the presence or absence of ecological limits.

\section{Counterarguments}

\section{Invasions and the Dynamics of Species Richness}

In many regions, species richness appears to have increased in recent decades as invasive species have colonized with few subsequent extinctions (Sax et al. 2002; Stohlgren et al. 2008). Such observations have been interpreted as evidence against saturation. As alluded to above, however, the term "saturation" confuses the idea of some hard limit to the number of species in a region as opposed to a stationary equilibrium reflecting the balance between opposing processes. If one result of the Anthropocene has been the increased spread of invasive species around the world, this effect amounts to an increase in background colonization or immigration rates (as in fig. $1 C$ ) and, hence, an expected increase in the equilibrial level of species richness. Human-facilitated dispersal of invasive species may also effectively increase the size of the regional species pool by allowing colonization from more distant locales than was possible in the past, again increasing the equilibrial richness value. As such, the observation that species richness has increased at particular sites in response to increased (humanmediated) colonization rates is fully consistent with a dynamic equilibrial model of species richness and cannot, by itself, reject the ecological limits hypothesis.

Something more difficult to ascertain is whether we are currently at the new equilibrium set by increased rates of human-assisted colonization or whether we are above it. Various authors have described the concept of "extinction debt," or the idea that there is often a time lag between when a system is perturbed and when species actually go extinct (Tilman et al. 1994; Jackson and Sax 2010). This implies that in some locales or regions, the observed increase in species richness may be temporary, with extinctions expected in the future. Two recent meta-analyses examining thousands of local communities over recent decades concluded that species richness has exhibited no directional trend on average (Vellend et al. 2013; Dornelas et al. 2014). These findings suggest that a balance between local colonizations and extinctions is the norm (Brown et al. 2001) and that invasions do not perpetually increase species richness.

\section{Phylogenetic Scale Matters}

Ecological limits based on an overarching energetic constraint will typically apply to large inclusive clades over which a zero-sum game is a reasonable characterization (Hurlbert and Stegen 2014b). The examination of small clades, such as genera or families, is expected to reveal a stronger signature of history, time, and the idiosyncrasy of specific resource-use strategies or climatic tolerances. Thus, individual mammal clades appear to show largely nonequilibrial dynamics at fine phylogenetic scales (Quental and Marshall 2013), but mammals as a whole (and more inclusive subgroups) show much greater evidence for equilibrial dynamics across the Cenozoic (Alroy 2009). A related prediction is that the species richness of large, inclusive clades should be strongly correlated with total resource availability, while small clades might show variable and even negative relationships (Currie 1991; Hurlbert and Stegen 2014b).

\section{Local-Regional Richness Correlations}

One argument against the idea of ecological limits at local scales is the observation that local richness often increases linearly with the richness of the broader regional species pool (Cornell and Lawton 1992; Karlson et al. 2004). If limits exist, so the argument goes, then local richness should level off as regional richness increases. However, an effect of regional richness on local communities is fully consistent with an equilibrial diversity framework, in that regions with a larger species pool should have immigration curves and, consequently, equilibrial richness values shifted toward higher values (MacArthur and Wilson 1963; Loreau and Mouquet 1999; He et al. 2005; fig. 1C). Second, the examination of this relationship in isolation ignores the potentially confounding effects of environmental variables that might drive both regional and local richness (White and Hurlbert 2010; Gronroos and Heino 2012). Finally, a number of statistical and conceptual problems have been raised regarding the connection between the local-regional richness relationship and inferences about limits (Srivastasa 1999; Hillebrand and Blenckner 2002; He et al. 2005).

\section{Dynamics of Local Communities Are (Largely) Irrelevant}

At the biogeographic scales discussed here, the ecological limits hypothesis does not make any claims about the equilibrium or nonequilibrium nature of local communities, as measured over ecological timescales. If all local communities are at equilibrium, it necessarily follows that regional biotas must also be at equilibrium because diversity at the regional scale is the sum of local richness. However, species richness at regional scales can show equilibrium properties even if local communities typically appear to be open, dynamic assemblages of species (DeAngelis and Waterhouse 1987; Turner et al. 1993; McPeek 2007). This idea is fundamental to metacommunity theory (Mouquet and Loreau 2003; Leibold et al. 2004) as well as more general theories of species richness that describe how environmental hetero- 
geneity and life-history trade-offs contribute to the maintenance of species richness at larger spatial scales, despite nonequilibrium dynamics at small spatial scales (Hutchinson 1951; Andrewartha and Birch 1954; Huston 1979; Petraitis et al. 1989). For example, in McPeek's (2007, 2008) metacommunity simulation model, global-scale species richness achieved a dynamic speciation-extinction equilibrium, but community composition at the local scale was continually changing in response to colonizations and extinctions at the level of individual patches. Most naturalists would accept that ecological succession following disturbance is an important process in many communities, yet these nonequilibrium dynamics are fully compatible with fixed or equilibrial global species pools (Sousa 1979). We agree with Wiens (2011) that local community dynamics as measured across paleontological timescales are relevant to testing the ecological limits hypothesis, but such samples are useful precisely because they average out the short-term fluctuations in community composition that can be attributed to disturbance, succession, and other nonequilibrial processes. Equilibrium dynamics at continental scales do not require equilibrium dynamics at the local scale, and hence tests of local-scale equilibrium are largely uninformative with respect to the ecological limits hypothesis.

\section{Conclusion: An Equilibrial World, Most of the Time}

Any general theory of diversity at continental or provincial scales must address a core set of observations about the dynamics of species richness in space and time. These observations include the striking variation in species richness among geographic regions, the general stability of species richness over geological timescales, and the response of richness to both mass extinctions and resource pulses. We believe that the ecological limits hypothesis can account for these observations with considerably fewer parameters than alternative models that postulate a diversityindependent, nonequilibrial world. We have argued here that the data are most consistent with a "strong" version of the ELH, but we would agree that many other factors can influence species richness. It is almost certainly the case that provincial biotas include residual effects of historical factors, and we also expect that the phylogenetic makeup of biotas has a substantial effect on the dynamics of resource use that ultimately determine richness. Nevertheless, we argue that richness reflects an equilibrium between speciation and extinction imposed by the finite nature of resources and that resource limits are the most important determinant of that equilibrial level.

In our opinion, biologists face two prominent challenges in testing the ELH. The first challenge involves defining the appropriate temporal and spatial scale over which limits are expected to operate. For example, life across the Pha- nerozoic has undoubtedly become more diverse, suggesting to some that there is little evidence for constraints on species richness (Benton 2009). Yet this increase in diversity is fully consistent with the ELH: when diversity rises, it often does so in response to rare but transformative innovations that increase the flux of energy and materials through the biosphere (Vermeij 1995; Allmon and Martin 2014). Much evidence from the fossil record suggests that, at the scale of geological periods, species richness is governed by the equivalent of a macroevolutionary zero-sum game.

The second challenge arises from the intersection of multiple competing factors that can account for large-scale diversity gradients. For example, Fine and Ree (2006) demonstrated that the correlation between the geographic area of 11 biomes and their present-day tree diversity was relatively weak but that the time-integrated area of biomes had much greater explanatory power. However, biome age is also correlated with energy and productivity: high-latitude boreal regions are young and energy poor and have few species relative to their area. In this essay, we have argued provocatively for the ubiquity of ecological limits, but our field is likely to continue debating this question for some time in part because large-scale patterns are poorly replicated (e.g., there are only a few biogeographic provinces) and multiple factors covary systematically with respect to species richness.

For this perspective, we were asked to address the question, What would it take to change your mind? We feel that the strong version of the ecological limits hypothesis would be rejected if species richness at continental/provincial scales is ultimately found to correlate more strongly with historical factors such as biome age than with area and energy, once collinearity of other variables has been taken into account. Likewise, we would be convinced if the apparent "epochal steady state" (Rosenzweig 1975) of species richness in the fossil record is found to be illusory. Finally, clear predictions about speciation dynamics at macroevolutionary scales emerge from the ecological limits model. In a diversity-dependent world, species diversity should increase primarily in response to evolutionary innovations that facilitate novel patterns of resource use. The diversity-independent model, in contrast, predicts that species diversity should rise as lineages acquire innovations that promote lineage splitting. Under the ELH, splitting is not enough: without ecological divergence, splitting merely carves a fixed pool of resources into smaller per capita shares, leading to low persistence of diverged lineages over geological timescales. At the risk of grossly oversimplifying a complex topic, we find it striking that so many factors that are expected to increase the evolution of reproductive isolation between diverging lineages (e.g., lineage splitting) are, at best, weakly correlated with macroevolution- 
ary speciation dynamics (Kraaijeveld et al. 2011; Rabosky and Matute 2013), yet innovations that increase resource capacities are consistently associated with large-scale diversity increase. diversity. Proceedings of the Royal Society B: Biological Sciences 277:3437-3443.

Brayard, A., G. Escarguel, H. Bucher, C. Monnet, T. Bruhwiler, N. Goudemand, T. Galfetti, and J. Guex. 2009. Good genes and good luck: ammonoid diversity and the end-Permian mass extinction. Science 325:1118-1121.

\section{Acknowledgments}

We thank L. Harmon and S. Harrison for an engaging debate on this topic and T. Price for organizing this special event at the 2014 ASN conference in Asilomar. We thank P. Fine, T. Price, A. Rabosky, R. Ricklefs, D. Schluter, anc members of the Rabosky lab for comments on the manuscript. Both authors contributed equally to this work.

\section{Literature Cited}

$\rightarrow$ Allen, A. P., J. H. Brown, and J. F. Gillooly. 2002. Global biodiversity, biochemical kinetics, and the energetic-equivalence rule. Sciencr 297:1545-1548.

$\rightarrow$ Allen, A. P., J. F. Gillooly, V. M. Savage, and J. H. Brown. 2006. Kinetic effects of temperature on rates of genetic divergence and speciation. Proceedings of the National Academy of Sciences of the USA 103:9130-9135.

Allmon, W. D., and R. E. Martin. 2014. Seafood through time revisited: the Phanerozoic increase in marine trophic resources and it: macroevolutionary consequences. Paleobiology 402:255-286.

Alroy, J. 2009. Speciation and extinction in the fossil record of Nortl $\rightarrow$ American mammals. Pages 301-323 in R. Butlin, J. Bridle, and D. Schluter, eds. Speciation and patterns of diversity. Cambridge University Press, Cambridge.

$\rightarrow$. 2010a. Geographical, environmental, and intrinsic biotic controls on Phanerozoic marine diversification. Palaeontology 53: 1211-1235.

$\rightarrow$. 2010b. The shifting balance of diversity among major marine animal groups. Science 321:1191-1194.

Andrewartha, H. G., and L. C. Birch. 1954. The distribution anc abundance of animals. University of Chicago Press, Chicago.

$\rightarrow$ Badgley, C. 2010. Tectonics, topography, and mammalian diversity. Ecography 33:220-231.

$\rightarrow$ Bambach, R. K. 1977. Species richness in marine benthic habitats through the Phanerozoic. Paleobiology 3:152-167.

$\rightarrow$ Bambach, R. K., A. M. Bush, and D. H. Erwin. 2007. Autecology anc $\rightarrow$ the filling of ecospace: key metazoan radiations. Palaeontology 50: $1-22$.

$\rightarrow$ Barry, J. C., M. E. Morgan, L. J. Flynn, D. Pilbeam, A. K. Behrens $\rightarrow$ meyer, S. M. Raza, I. A. Khan, C. Badgley, J. Hicks, and J. Kelley. 2002. Faunal and environmental change in late Miocene Siwaliks of northern Pakistan. Paleobiology 28:1-71.

$\rightarrow$ Benton, M. J. 2009. The Red Queen and the Court Jester: species diversity and the role of biotic and abiotic factors through time. Science 323:728-732.

$\rightarrow$ Boyce, C. K., T. J. Brodribb, T. S. Feild, and M. A. Zwieniecki. 2009 Angiosperm leaf vein evolution was physiologically and environmentally transformative. Proceedings of the Royal Society B: Biological Sciences 276:1771-1776.

$\rightarrow$ Boyce, C. K., and J.-E. Lee. 2010. An exceptional role for flowering plant physiology in the expansion of tropical rainforests and bio- $\rightarrow$ Brodribb, T. J., and T. S. Feild. 2010. Leaf hydraulic evolution led to a surge in leaf photosynthetic capacity during early angiosperm diversification. Ecology Letters 13:175-183.

Brown, J. H., S. K. M. Ernest, J. M. Parody, and J. P. Haskell. 2001. Regulation of diversity: maintenance of species richness in changing environments. Oecologia (Berlin) 126:321-332.

Bush, A. M., and R. K. Bambach. 2011. Paleoecologic megatrends in marine Metazoa. Annual Review of Earth and Planetary Sciences 39:241-269.

$\rightarrow$ Chen, Z.-Q., and M. J. Benton. 2012. The timing and pattern of biotic recovery following the end-Permian mass extinction. Nature Geoscience 5:375-383. doi:10.1038/NGEO1475.

$\rightarrow$ Cornell, H. V. 2013. Is regional species diversity bounded or unbounded? Biological Reviews 88:140-165.

Cornell, H. V., and J. H. Lawton. 1992. Species interactions, local and regional processes, and limits to the richness of ecological communities - a theoretical perspective. Journal of Animal Ecology 61:1-12.

Coyne, J. A., and H. A. Orr. 2004. Speciation. Sinauer, Sunderland, MA.

$\rightarrow$ Cracraft, J. 1985. Biological diversity and its causes. Annals of the Missouri Botanical Garden 72:794-822.

Currie, D. J. 1991. Energy and large-scale patterns of animal and plant species richness. American Naturalist 137:27-49.

Cusimano, N., and S. S. Renner. 2010. Slowdowns in diversification rates from real phylogenies may not be real. Systematic Biology 59:458-464.

$\rightarrow$ DeAngelis, D. L., and J. C. Waterhouse. 1987. Equilibrium and nonequilibrium concepts in ecological models. Ecological Monographs 57:1-21.

$\rightarrow$ Dornelas, M., N. J. Gotelli, B. McGill, H. Shimadzu, F. Moyes, C. Sievers, and A. E. Magurran. 2014. Assemblage time series reveal biodiversity change but not systematic loss. Science 344:296-299.

$\rightarrow$ Erwin, D. H. 2001. Lessons from the past: biotic recoveries from mass extinctions. Proceedings of the National Academy of Sciences of the USA 98:5399-5403.

$\rightarrow$ Erwin, D. H., J. W. Valentine, and J. J. Sepkoski. 1987. A comparativestudy of diversification events: the early Paleozoic versus the Mesozoic. Evolution 41:1177-1186.

Etienne, R. S., and B. Haegeman. 2012. A conceptual and statistical framework for adaptive radiations with a key role for diversity dependence. American Naturalist 180:E75-E89.

Etienne, R. S., and J. Rosindell. 2012. Prolonging the past counteracts the pull of the present: protracted speciation can explain observed slowdowns in diversification. Systematic Biology 61:204-213.

$\rightarrow$ Ezard, T. H. G., T. Aze, P. N. Pearson, and A. Purvis. 2011. Interplay between changing climate and species' ecology drives macroevolutionary dynamics. Science 332:349-351.

$\rightarrow$ Feild, T. S., T. J. Brodribb, A. Iglesias, D. S. Chatelet, A. Baresch, G. R. J. Upchurch, B. Gomez, et al. 2011. Fossil evidence for Cretaceous escalation in angiosperm leaf venation. Proceedings of the National Academy of Sciences of the USA 108:8363-8366.

$\rightarrow$ Fine, P. V. A., and R. H. Ree. 2006. Evidence for a time-integrated species-area effect on the latitudinal gradient in tree diversity. American Naturalist 168:796-804. 
$\rightarrow$ Fischer, A. G. 1960. Latitudinal variations in organic diversity. Evo $\rightarrow$ lution 14:64-81.

$\rightarrow$ Gillooly, J. F., and A. P. Allen. 2007. Linking global patterns in biodiversity to evolutionary dynamics using metabolic theory. Ecol ogy 88:1890-1894.

$\rightarrow$ Gilman, L. N., and S. D. Wright. 2006. The influence of productivity on the species richness of plants: a critical assessment. Ecology 87 1234-1243.

$\rightarrow$ Gronroos, M., and J. Heino. 2012. Species richness at the guild level: effects of species pool and local environmental conditions on stream macroinvertebrate communities. Journal of Animal Ecolog: 81:679-691.

$\rightarrow$ Harmon, L. J., and S. Harrison. 2015. Species diversity is dynamic and unbounded at local and continental scales. American Natural ist 185:584-593.

$\rightarrow$ Harnik, P. G., C. Simpson, and J. L. Payne. 2012. Long-term differences in extinction risk among the seven forms of rarity. Proceed ings of the Royal Society B: Biological Sciences 279:4969-4976.

$\rightarrow$ He, F., K. J. Gaston, E. F. Connor, and D. S. Srivastasa. 2005. The local-regional relationship: immigration, extinction, and scale. Ecol $\rightarrow$ ogy 86:360-365.

$\rightarrow$ Hillebrand, H., and T. Blenckner. 2002. Regional and local impact on species diversity - from pattern to process. Oecologia (Berlin) 132: 479-491.

Hubbell, S. P. 2001. The unified neutral theory of biodiversity and biogeography. Princeton University Press, Princeton, NJ.

$\rightarrow$ Hurlbert, A. H., and J. C. Stegen. 2014a. On the processes generating latitudinal richness gradients: identifying diagnostic patterns and predictions. Frontiers in Genetics 5:420.

$\rightarrow-2014 b$. When should species richness be energy limited, anc how would we know? Ecology Letters 17:401-413.

$\rightarrow$ Huston, M. A. 1979. A general hypothesis of species diversity. American Naturalist 113:81-101.

$\rightarrow$ Hutchinson, G. E. 1951. Copepodology for the ornithologist. Ecology 32:571-577.

$\rightarrow$ Jackson, S. T., and D. F. Sax. 2010. Balancing biodiversity in a chang ing environment: extinction debt, immigration credit and specie turnover. Trends in Ecology and Evolution 25:153-160.

$\rightarrow$ Jan de Boer, H., M. B. Eppinga, M. J. Wassen, and S. Dekker. 2012. A critical transition in leaf evolution facilitated the Cretaceous an giosperm revolution. Nature Communications 3:1221.

$\rightarrow$ Jaramillo, C., M. J. Rueda, and G. Mora. 2006. Cenozoic plant diver $\rightarrow$ sity in the Neotropics. Science 311:1893-1896.

$\rightarrow$ Jetz, W., and P. V. A. Fine. 2012. Global gradients in vertebrate di $\rightarrow$ versity predicted by historical area-productivity dynamics and contemporary environment. PLoS Biology 10:e1001292.

$\rightarrow$ Karlson, R. H., H. V. Cornell, and T. P. Hughes. 2004. Coral communities are regionally enriched along an oceanic biodiversity gradi ent. Nature 429:867-870.

$\rightarrow$ Kisel, Y., and T. G. Barraclough. 2010. Speciation has a spatial scale that depends on levels of gene flow. American Naturalist 175:316-334.

Knoll, A. H. 1986. Patterns of change in plant communities through geological time. Pages 126-141 in J. Diamond and T. J. Case, eds. Community ecology. Harper \& Row, New York.

$\rightarrow$ Kraaijeveld, K., F. J. L. Kraaijeveld-Smit, and M. E. Maan. 2011. Sexual selection and speciation: the comparative evidence revisited. Biological Reviews 86:367-377.

$\rightarrow$ Kreft, H., and W. Jetz. 2007. Global patterns and determinants of vascular plant diversity. Proceedings of the National Academy of Sciences of the USA 104:5925-5930.
Krug, A. Z., and M. E. Patzkowsky. 2004. Rapid recovery from the Late Ordovician mass extinction. Proceedings of the National Academy of Sciences of the USA 101:17605-17610.

Leibold, M. A., M. Holyoak, N. Mouquet, P. Amarasekare, J. M. Chase, M. F. Hoopes, R. D. Holt, et al. 2004. The metacommunity concept: a framework for multi-scale community ecology. Ecology Letters 7:601-613.

$\rightarrow$ Lidgard, S., and P. R. Crane. 1990. Angiosperm diversification and Cretaceous floristic trends: a comparison of palynofloras and leaf macrofloras. Paleobiology 16:77-93.

Liow, L. H., and J. A. Finarelli. 2014. A dynamic global equilibrium in carnivoran diversification over 20 million years. Proceedings of the Royal Society B: Biological Sciences 281:20132312.

Liow, L. H., T. B. Quental, and C. R. Marshall. 2010. When can decreasing diversification rates be detected with molecular phylogenies and the fossil record? Systematic Biology 59:646-659.

Loreau, M., and N. Mouquet. 1999. Immigration and the maintenance of local species diversity. American Naturalist 154:427440.

Lupia, R., S. Lidgard, and P. R. Crane. 1999. Comparing palynological abundance and diversity: implications for biotic replacement during the Cretaceous angiosperm radiation. Paleobiology 25:305340 .

$\rightarrow$ MacArthur, R. H. 1969. Patterns of communities in the tropics. Biological Journal of the Linnean Society 1:19-30.

$\rightarrow$ MacArthur, R. H., and E. O. Wilson. 1963. An equilibrium theory of insular zoogeography. Evolution 17:373-387.

- 1967. The theory of island biogeography. Princeton University Press, Princeton, NJ.

$\rightarrow$ Manne, L. L., T. M. Brooks, and S. L. Pimm. 1999. Relative risk of extinction of passerine birds on continents and islands. Nature 399:258-261.

Mayr, E. 1963. Animal species and evolution. Belknap, Cambridge, MA.

$\rightarrow$ McPeek, M. A. 2007. The macroevolutionary consequences of ecological differences among species. Palaeontology 50:111-129.

Mitter, C., B. Farrell, and B. Wiegmann. 1988. The phylogenetic study of adaptive zones: has phytophagy promoted insect diversification? American Naturalist 132:107-128.

Moen, D. S., and H. Morlon. 2014. Why does diversification slow down? Trends in Ecology and Evolution 29:190-197.

$\rightarrow$ Mouquet, N., and M. Loreau. 2003. Community patterns in sourcesink metacommunities. American Naturalist 162:544-557.

Payne, J. L., and S. Finnegan. 2007. The effect of geographic range on extinction risk during background and mass extinction. Proceedings of the National Academy of Sciences of the USA 104:1050610511.

Peters, S. E. 2005. Geologic constraints on the macroevolutionary history of marine animals. Proceedings of the National Academy of Sciences of the USA 102:12326-12331.

$\rightarrow$ Petraitis, P. S., R. E. Latham, and R. A. Niesenbaum. 1989. The maintenance of species diversity by disturbance. Quarterly Review of Biology 64:393-418.

$\rightarrow$ Pigot, A. L., and J. A. Tobias. 2013. Species interactions constrain geographic range expansion over evolutionary time. Ecology Letters 16:330-338.

Price, T. D. 2008. Speciation in birds. Roberts, Greenwood Village, CO.

2010. The roles of time and ecology in the continental radiation of the Old World leaf warblers (Phylloscopus and Seicercus). 
Philosophical Transactions of the Royal Society B: Biological Sci $\rightarrow$ Sexton, J. P., P. J. McIntyre, A. L. Angert, and K. J. Rice. 2009. Evoences 365:1749-1762.

$\rightarrow$ Price, T. D., and M. Kirkpatrick. 2009. Evolutionarily stable range limits set by interspecific competition. Proceedings of the Roya Society B: Biological Sciences 276:1429-1434.

$\rightarrow$ Quental, T. B., and C. R. Marshall. 2013. How the Red Queen drives terrestrial mammals to extinction. Science 341:290-292.

$\rightarrow$ Rabosky, D. L. 2009. Heritability of extinction rates links diversification patterns in molecular phylogenies and fossils. Systematic Biology 58:629-640.

$\rightarrow$. 2012. Testing the time-for-speciation effect in the assem bly of regional biotas. Methods in Ecology and Evolution 3:224233.

$\rightarrow$. 2013. Diversity dependence, ecological speciation, and the role of competition in macroevolution. Annual Review of Ecology, Evolution, and Systematics 44:481-502. doi:10.1146/annurev-ecolsy: $-110512-13580$. lution and ecology of species range limits. Annual Review of Ecology, Evolution, and Systematics 40:415-436.

Sousa, W. P. 1979. Disturbance in marine intertidal boulder fields: the nonequilibrium maintenance of diversity. Ecology 60:12251239 .

$\rightarrow$ Srivastasa, D. S. 1999. Using local-regional richness plots to test for species saturation: pitfalls and potentials. Journal of Animal Ecology $68: 1-16$.

$\rightarrow$ Stephens, P. R., and J. J. Wiens. 2003. Explaining species richness from continents to communities: the time-for-speciation effect in emydid turtles. American Naturalist 161:112-128.

$\rightarrow$ Stohlgren, T. J., D. T. Barnett, C. S. Jarnevich, C. Flather, and J. Kartesz. 2008. The myth of plant species saturation. Ecology Letters 11:313-326.

$\rightarrow$ Tilman, D., R. M. May, C. L. Lehman, and M. A. Nowak. 1994. Habitat destruction and the extinction debt. Nature 371:65-66.

$\rightarrow$ Rabosky, D. L., and I. J. Lovette. 2008. Density-dependent diversifi cation in North American wood warblers. Proceedings of the Royal Society B: Biological Sciences 275:2363-2371.

$\rightarrow$ Rabosky, D. L., and D. R. Matute. 2013. Macroevolutionary speciation rates are decoupled from the evolution of intrinsic reproductive isolation in Drosophila and birds. Proceedings of the National Academy of Sciences of the USA 110:15354-15359.

$\rightarrow$ Revell, L. J., L. J. Harmon, and R. E. Glor. 2005. Underparameterized model of sequence evolution leads to bias in the estimation of diversification rates from molecular phylogenies. Systematic Biology 54:973-983.

$\rightarrow$ Ricklefs, R. E., and E. Bermingham. 2007. The causes of evolutionary radiations in archipelagoes: passerine birds in the lesser Antilles. American Naturalist 169:285-297.

$\rightarrow$ Rohde, K. 1992. Latitudinal gradients in species diversity: the search for the primary cause. Oikos 65:514-527.

Rosenzweig, M. L. 1975. On continental steady states of species di versity. Pages 121-140 in M. L. Cody and J. M. Diamond, eds Ecology and evolution of communities. Belknap, Cambridge, MA . 1995. Species diversity in space and time. Cambridge University Press, Cambridge.

Turner, M. G., W. H. Romme, R. H. Gardner, R. V. O’Neill, and T. K. Kratz. 1993. A revised concept of landscape equilibrium: disturbance and stability on scaled landscapes. Landscape Ecology 8:213-227.

Van Valen, L. 1976. Energy and evolution. Evolutionary Theory 1:179-229.

Van Valkenburgh, B., and C. M. Janis. 1993. Historical diversity patterns in large mammalian herbivores and carnivores. Pages 330-340 in R. E. Ricklefs and D. Schluter, eds. Species diversity in ecological communities: historical and geographical perspectives. University of Chicago Press, Chicago.

Vellend, M., L. Taeten, I. H. Myers-Smith, S. C. Elmendorf, R. Beausejour, C. D. Brown, P. De Frenne, K. Verheyen, and S. Wipf. 2013. Global meta-analysis reveals no net change in local-scale plant biodiversity over time. Proceedings of the National Academy of Sciences of the USA 110:19456-19459.

Vermeij, G. J. 1995. Economics, volcanos, and Phanerozoic revolutions. Paleobiology 21:125-152.

$\rightarrow$ Vermeij, G. J., and P. D. Roopnarine. 2013. Reining in the Red Queen: the dynamics of adaptation and extinction reexamined. Paleobiology 39:560-575.

Rosenzweig, M. L., F. Drumlevitch, K. L. Borgmann, A. D. Flesch $\rightarrow$ Wagner, P. J., M. A. Kosnik, and S. Lidgard. 2006. Abundance disS. M. Grajeda, G. D. Johnson, K. Mackay, et al. 2012. An ecological telescope to view future terrestrial vertebrate diversity. Evolutionary Ecology Research 14:247-268.

$\rightarrow$ Rosenzweig, M. L., and J. A. Taylor. 1980. Speciation and diversity in Ordovician invertebrates: filling niches quickly and carefully. Oikos 35:236-243.

$\rightarrow$ Sallan, L. C., and M. I. Coates. 2010. End-Devonian extinction and a bottleneck in the early evolution of modern jawed vertebrates. Proceedings of the National Academy of Sciences of the USA 107 10131-10135.

$\rightarrow$ Sax, D. F., S. D. Gaines, and J. H. Brown. 2002. Species invasions ex ceed extinctions on islands worldwide: a comparative study o plants and birds. American Naturalist 160:766-783.

$\rightarrow$ Sepkoski, J. J. 1978. A kinetic model of Phanerozoic taxonomic diversity. I. Analysis of marine orders. Paleobiology 4:223-251. tributions imply elevated complexity of post-Paleozoic marine ecosystems. Science 314:1289-1292.

$\rightarrow$ White, E. P., and A. H. Hurlbert. 2010. The combined influence of local environment and regional enrichment on bird species richness. American Naturalist 175:E35-E43.

$\rightarrow$ Wiens, J. J. 2011. The causes of species richness patterns across space, time, and clades and the role of "ecological limits." Quarterly Review of Biology 86:75-96.

Wing, S. L., and W. A. DiMichele. 1995. Conflict between global and local changes in plant diversity through geological time. Palaios 10:551-564.

Wright, D. H. 1983. Species-energy theory: an extension of speciesarea theory. Oikos 41:496-506. 